\title{
MODELING SPACECRAFT OSCILLATIONS IN HRSC IMAGES OF MARS EXPRESS
}

\author{
J. Bostelmann, C. Heipke \\ Institute of Photogrammetry and GeoInformation (IPI) \\ Leibniz Universität Hannover, Germany \\ Nienburger Str.1, D-30167 Hannover \\ bostelmann@ipi.uni-hannover.de, heipke@ipi.uni-hannover.de \\ http://www.ipi.uni-hannover.de/mars.html
}

KEY WORDS: Bundle Adjustment, Multi-Line Camera, HRSC, Planetary Imagery

\begin{abstract}
:
Since January 2004 the High Resolution Stereo Camera (HRSC) is mapping planet Mars. The multi-line sensor on board the ESA Mission Mars Express images the Martian surface with a resolution of up to $12 \mathrm{~m}$ per pixel in three dimensions and in color. As part of the Photogrammetric/Cartographic Working Group of the HRSC Science Team the Institute of Photogrammetry and GeoInformation (IPI) of the Leibniz Universität Hannover is involved in photogrammetrically processing the HRSC image data. To derive high quality 3D surface models, color orthoimages or other products, the accuracy of the observed position and attitude information in many cases should be improved. This is carried out via a bundle adjustment. In a considerable number of orbits the results of the bundle adjustment are disturbed by high frequency oscillations. This paper describes the impact of the high frequency angular spacecraft movement to the processing results of the last seven years of image acquisition and how the quality of the HRSC data products is significantly improved by modeling these oscillations.
\end{abstract}

\section{INTRODUCTION}

The Mars Express mission conducted by the European Space Agency (ESA) is investigating the Red Planet since January 2004. One of the scientific instruments on board the orbiter is the High Resolution Stereo Camera (HRSC). This multi-line stereo scanner is equipped with 5 panchromatic and 4 color charge-coupled device (CCD) line detectors, each with 5176 pixels. The configuration of the sensor lines on the focal plane of the camera allows a stereoscopic image acquisition of the Martian surface (Neukum et al., 2004).

By simultaneously reading out (up to) nine CCD sensor lines, while the camera points at Mars when reaching the periapsis of the highly elliptical orbit, long image strips are created. Because of the different viewing angles in the images of these strips, it is possible to obtain stereo color data, with a resolution of up to $12 m$ per pixel, depending on the flying height.

To use the HRSC image data for the derivation of digital terrain models (DTMs) or orthoimages the knowledge about the position and attitude of the camera is essential. The position is observed via Doppler measurements, and star trackers control the attitude of the camera. The combination of these observations is called the nominal exterior orientation of the camera. In many cases the quality of the exterior orientation is not accurate enough for a precise photogrammetric point determination. Therefore, a large number of automatically determined tie points is used in a bundle adjustment to improve the accuracy of the exterior orientation and to exploit the full potential of the stereo image data.

The basic theory of a combined bundle adjustment for HRSC imagery and the MOLA DTM is described in chapter 2 . In chapter 3 recent results of the operational standard processing carried out at the Institute of Photogrammetry and GeoInformation (IPI) of Leibniz Universität Hannover are presented. A closer look at the orbits, where high frequent oscillations distort the results, is taken in chapter 4 including a solution to the problem. Conclusions are drawn in the last chapter.

\section{BUNDLE ADJUSTMENT}

In this section the concept of the bundle adjustment for HRSC imagery is described. It is based on the well known approach used in photogrammetry for aerial triangulation, by which the exterior orientation is simultaneously determined for all images used in the bundle adjustment. In case of HRSC the images are not taken at separate points but in a continuous motion. Thus, the exterior orientation must be modeled along the spacecrafts trajectory as a function of time. Almost three decades ago the concept of orientation points was proposed to solve this problem (Hofmann et al., 1982). Today it is a common approach in the processing of multi-line sensor data.

To fit the photogrammetrically determined 3D coordinates of a photogrammetric bundle block to an regional or global reference system, an adequate number of ground control points is normally used in aerial or spaceborne photogrammetry. For areas without ground control points the usage of available digital terrain models (DTMs) is possible to obtain an absolute fit (Strunz, 1993). On Mars the MOLA DTM provides the best accuracy. Therefore, a combined bundle adjustment for HRSC image data and the MOLA DTM as control information was developed, implemented and tested (Spiegel, 2007b).

\subsection{Mathematical model}

The general approach of the bundle adjustment is a nonlinear least-squares adjustment. This optimization aims to find the best set of unknown model parameters to explain the observations, so that the sum of squared weighted residuals (1) is minimized.

$$
\sum v^{T} W v
$$

To take the accuracy information of the observations into account the residuals $v$ are weighted by the inverse cofactor matrix of the observations $\left(W=C^{-1}\right)$.

The residuals $v$ are given by the differences between the actual 
observations $z$ and a function of the unknown parameters $U$ modeling the corresponding observation.

$$
v=f(U)-z
$$

For a combined adjustment of HRSC images and the MOLA DTM there are four types of observations, which have to be modeled: image coordinates, orientation parameters, free unknowns and DTM conditions. Hence there are four types of observation equations, described in the following sections.

\subsection{Extended collinearity equations}

In photogrammetry the central perspective model is often used to describe the physical process of imaging. The fact that an image point, the perspective center of the camera and the corresponding object point lie on a straight line is modeled by the collinearity equations. For multi-line cameras an extension was proposed (Hofmann et al., 1982). This concept of the extended collinearity equations was later improved and adapted to space missions such as MOMS-02/D2, MOMS-P2, Mars 96 and HRSC on Mars Express (Müller, 1991), (Dorrer et al., 1995), (Ohlhof, 1996), (Kornus, 1999), (Spiegel, 2007b).

For the image coordinates $z_{x y}$ the nonlinear observation equations are

$$
v_{x y}=f_{x y}\left(U_{P}, U_{O}(t)\right)-z_{x y}
$$

where $f_{x y}$ substitutes the classical collinearity equations (with constant interior orientation parameters). $U_{P}=(X, Y, Z)^{T}$ are the coordinates of the corresponding object point in a $3 \mathrm{D}$ coordinate system and $U_{O}=(X, Y, Z, \varphi, \omega, \kappa)^{T}$ describes the exterior orientation of the camera. Because HRSC is a line-scanner the parameters of exterior orientation are a function of time. The orientation parameters at the time $t$ are interpolated by piecewise cubic Lagrange polynomials.

$$
\begin{aligned}
& U_{O}(t)=U_{B}+U_{D}\left(t-t_{0}\right)+ \\
& +\sum_{i=m-1}^{m+2} U_{O}\left(t_{i}\right) \prod_{j=m-1, j \neq i}^{m+2} \frac{t-t_{j}}{t_{i}-t_{j}}+\delta U_{O}
\end{aligned}
$$

The time $t$, when the image line containing the point was taken, lies between the two points in time $t_{m}, t_{m+1}$ of the previous and the next orientation point.

$$
t_{m} \geq t \geq t_{m+1}
$$

To consider systematic errors in observed exterior orientation like bias or drift (see section 2.3), the parameters $U_{B}$ and $U_{D}$ are added. $\delta U_{O}$ is introduced to avoid interpolation errors and is calculated by subtracting the values of the nominal exterior orientation from the interpolated values for every image line. The $\delta U_{O}$ are used as constant values during the adjustment. Finally, the distance between $t_{m}$ and $t_{m+1}$ is defined by the orientation point distance (OPD).

$$
t_{O P D}=t_{m+1}-t_{m}
$$

When using 3rd order Lagrange polynomials a sequence of four groups of orientation parameters is used to describe the exterior orientation $U_{m}$ at the orientation point $m$.

$$
U_{m}=\left(U_{O}\left(t_{m-1}\right), U_{O}\left(t_{m}\right), U_{O}\left(t_{m+1}\right), U_{O}\left(t_{m+2}\right)\right)
$$

In summary the exterior orientation is a function of time, bias, drift, and a set of orientation parameters.

$$
U_{O}(t)=U_{O}\left(t, U_{B}, U_{D}, U_{m}\right)
$$

\subsection{Observed orientation parameters}

The second kind of observation equations is needed to introduce observed values for the orientation parameters at the orientation points into the adjustment. At an orientation point $m$ the nominal exterior orientation can be used directly for the six elements of the exterior orientation $z_{m}=\left(X_{m}, Y_{m}, Z_{m}, \varphi_{m}, \omega_{m}, \kappa_{m}\right)^{T}$. Hence the observation equations for the observed exterior orientation are

$$
v_{m}=f_{m}\left(U_{B}, U_{D}, U_{O}\left(t_{m}\right)\right)-z_{m}
$$

where $f_{m}$ describes the direct relation between the observations $z_{m}$ and the unknown orientation parameters $U_{O}\left(t_{m}\right)$ with simultaneous consideration of bias $U_{B}$ and drift $U_{D}$.

$$
v_{m}=U_{B}+U_{D}\left(t_{m}-t_{0}\right)+U_{O}\left(t_{m}\right)-z_{m}
$$

\subsection{Free unknowns}

To introduce the free unknown parameters for bias $U_{B}$ and drift $U_{D}$ into the functional and stochastic model of the adjustment, another set of observation equations is needed. These equations allow the modeling of systematic effects for every element of the observed orientation parameters.

$$
v_{B}=f_{B}\left(U_{B}\right)-z_{B}=U_{B}-z_{B}
$$

$$
v_{D}=f_{D}\left(U_{D}\right)-z_{D}=U_{D}-z_{D}
$$

Here $f_{B}$ and $f_{D}$ describe a direct relation between observations and unknown parameters.

\subsection{Control information}

For adding control information into the bundle adjustment a fourth kind of observation equation is used

$$
v_{h}=f_{h}\left(U_{P}\right)-z_{h}
$$

where $v_{h}$ is the residual of observed height differences $z_{h}$ and modeled height differences $f_{h}\left(U_{P}\right)$. If $v_{h}$ describes only the height differences between the MOLA surface and the photogrammetrically determined height $Z_{P}$, the MOLA surface height depends on planimetric coordinates $X_{P}$ and $Y_{P}$, and thus we would end up with a condition equation of a Gauss-Helmert model rather than an observation equation of the Gauss-Markov model, in which we can introduce a standard deviation for the observations. 
Considering that HRSC object points and the MOLA DTM describe the same physical surface, the observations of the height difference should always be zero $\left(z_{h}=0\right) . f_{h}$ models these height differences as a function of the unknown object point coordinates $\left(X_{P}, Y_{P}, Z_{P}\right)$. The height of every HRSC object point $Z_{P}$ is subtracted from the height of a bilinear surface defined by a regular grid of the MOLA DTM. The 3D coordinates of the four MOLA grid points $\left(X_{i}, Y_{i}, Z_{i}\right)$ are used as constant values and $d$ defines their grid width.

$$
f_{h}=Z_{P}-\sum_{i=1}^{4}\left(1-\frac{X_{P}-X_{i}}{d}\right)\left(1-\frac{Y_{P}-Y_{i}}{d}\right) Z_{i}
$$

\subsection{Stochastic model}

The accuracy of all observations described above is considered by the weight matrix $W$ (see equation 1 ). Thus every observation is weighted by its a priori standard deviation $\sigma_{0}$. This also allows to use observations as constants with $\sigma_{0}=0$ or as free unknowns with $\sigma_{0} \rightarrow \infty$.

The results presented in the next chapter, are obtained by using an adjustment concept specially developed for the HRSC data (Spiegel, 2007b). It uses the standard deviations given in table 1.

\begin{tabular}{|l|l|}
\hline Observations & $\sigma$ a priori \\
\hline \hline image coordinates & $\sigma_{0, x y}=1 \mu \mathrm{m}$ \\
\hline \hline orientation parameters, position & $\sigma_{0, X Y Z}=0.01 \mathrm{~m}$ \\
\hline position bias & $\sigma_{0, X Y Z}=1000 \mathrm{~m}$ \\
\hline position drift & $\sigma_{0, Z}=0.01 \mathrm{~m}$ \\
\hline \hline orientation parameters, angles & $\sigma_{0, \varphi \omega \kappa}=0.028 \mathrm{gon}$ \\
\hline angular bias & $\sigma_{0, \varphi \omega \kappa}=0$ \\
\hline angular drift & $\sigma_{0, \varphi \omega \kappa}=0$ \\
\hline \hline MOLA DTM & $\sigma_{0, D T M}=100 \mathrm{~m}$ \\
\hline
\end{tabular}

Table 1: A priori accuracy of observations used in the bundle adjustment

The concept for the bundle adjustment includes an outliers detection and recommends an orientation point distance of $O P D=$ $10 s$.

\section{PROCESSING RESULTS}

\subsection{HRSC data}

Based on the bundle adjustment explained in chapter 2 the method for operational determination of tie points (Schmidt et al., 2008) and the concept of bundle adjustment (Spiegel, 2007a) has been combined in an automatic processing work flow for the systematic adjustment of the exterior orientation of HRSC image data.

Hence it was possible to process all available data of the HRSC on a computer cluster at the RRZN (Regionales Rechenzentrum für Niedersachsen) in a reasonable time. Because both, the determination and matching of the tie points and the process of bundle adjustment are very CPU time and memory consuming, the data was processed in parallel on up to $200 \mathrm{CPUs}$ of the cluster.

The processed data contain the images from orbits recorded from the moment the Mars Express mission entered into orbit around the planet in January 2004 until November 2010. In this time of almost seven years the Mars Express mission has surrounded the planet 8761 times. In about 3000 of these orbits the camera took imagery theoretically suitable for stereo processing. A successful bundle adjustment and registration to the MOLA DTM, as described in chapter 2, was obtained for the HRSC imagery of 2535 orbits. Reasons for a failure of the automatic processing work flow are mostly an insufficient number of tie points found by automatic image matching, because of bad image quality.

\subsection{Mean intersection error}

To evaluate the quality of the exterior orientation data before and after the bundle adjustment for every orbit, the mean intersection error is used. The mean intersection error summarizes the accuracy of all object points used for the generation of a DTM (Gwinner et al., 2009). Because the matching procedure and the other input data, as the images and the calibration data (interior orientation) of the HRSC sensor are constant, the mean intersection error only depends on the accuracy of the exterior orientation data. Therefore, it can be used to reveal the improvement of the exterior orientation by bundle adjustment.

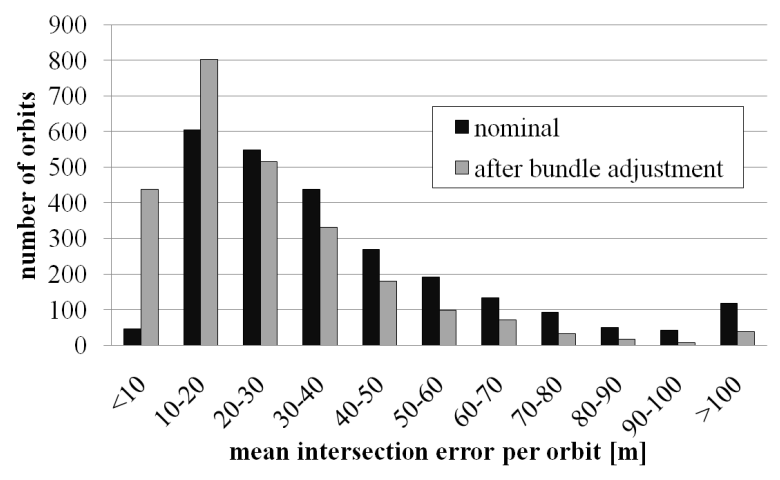

a) histogram

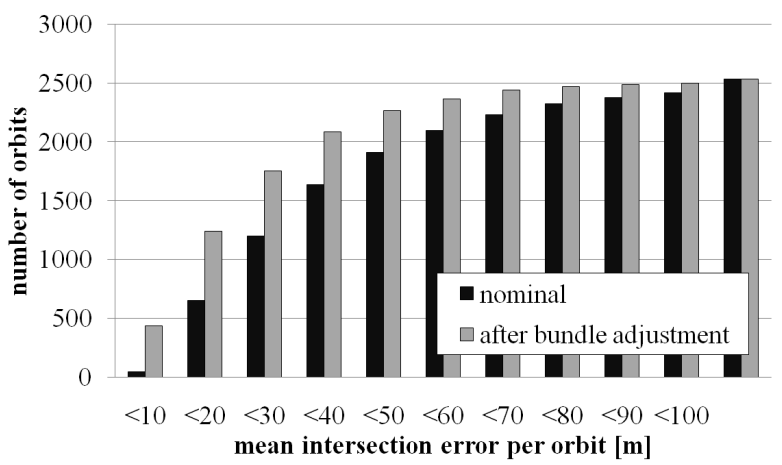

b) cumulative histogram

Figure 1: Number of orbits grouped by their mean intersection error before (black) and after (gray) the improvement of the exterior orientation by bundle adjustment

In Figure 1 the mean intersection error for a total of 2535 successfully processed orbits is presented. The orbits are classified according to their intersection error in ranges of $10 \mathrm{~m}$. The black bars represent the quality of the exterior orientation data before, and the gray bars after the process of bundle adjustment. Figure 1a shows the histogram of mean intersection errors, in Figure $1 \mathrm{~b}$ the cumulative histogram is depicted. It is shown that the number of orbits with a mean intersection error $<10 \mathrm{~m}$ distinctly increases after the bundle adjustment (more precisely from 47 to 437). Also the quantity of orbits with a mean intersection error lying between $10 \mathrm{~m}$ and $20 \mathrm{~m}$ increases by a number of 200 . On the other hand the number of orbits with higher errors obviously 
decreases. E.g. the number with an error $>100 \mathrm{~m}$ is reduced from 119 to 38 orbits. Probably because there was no exclusion of orbits with obvious image degradation, the mean intersection errors presented here are somewhat higher than in other statistics of HRSC DTM processing (e.g. Gwinner et al., 2010). In general the mean intersection error presented in this paper should not be taken as an absolute criterion for the quality of HRSC data, but it is a good indicator for a relative improvement.

Through bundle adjustment the mean intersection errors of individual orbits are reduced by an average factor of 1.7. It should be noted, that for a number of 42 orbits $(1.6 \%)$ the standard adjustment process is not successful, leading to a significant reduction of the quality. These orbits are not included in the statistics of the successfully processed 2535 orbits. Table 2 breaks down the findings into five groups. In about a tenth of all cases $(8.1 \%)$ the adjustment of the exterior orientation causes only minor variations in the quality of the generated points. However for 559 orbits $(22.1 \%)$ the accuracy of the object points could be improved by a factor of 2 or more.

\begin{tabular}{|c||c|c|}
\hline Improvement & Nr. of Orbits & \% of 2535 \\
\hline \hline $0.95-1.05$ & 206 & $8.1 \%$ \\
\hline $1.05-1.50$ & 1192 & $47.0 \%$ \\
\hline $1.50-2.00$ & 578 & $22.8 \%$ \\
\hline $2.00-4.00$ & 503 & $19.8 \%$ \\
\hline$>4.00$ & 56 & $2.2 \%$ \\
\hline
\end{tabular}

Table 2: Factor of reduction of the mean intersection error

Another advantage of the bundle adjustment is the absolute positioning of the object points by fitting them to the MOLA DTM. This has been shown in detail for a test dataset of 45 orbits (Schmidt et al., 2008).

To process larger regional or even a global block, it is advantageous to increase the number of orbits with good single strip processing results. In this spirit the adjusted exterior orientation is used as input data for the simultaneous adjustment of image strips combined to regional blocks and the derivation of DTM and orthoimage-mosaics (Dumke et al., 2008).

For further analysis of the adjustment results color coded DTM point accuracy maps, which are based on the intersection error described above, were generated for every orbit before and after the bundle adjustment. Following this the quality map of every orbits was visually inspected for remaining systematic errors.

\section{OSCILLATIONS IN ORIENTATION DATA}

In some orbits the maps of DTM point accuracy revealed obvious systematic effects (Scholten, personal communication, 2008). The quality of the DTM points periodically increases and decreases along the strip in the flight direction of the camera. The first orbit where this oscillation has been found in a severe form was $\mathrm{h} 2028$. In the data processed so far (h0008-h8761) a number of 299 orbits ( $12 \%$ of the 2535 orbits) is affected.

The causes for this variations in the quality of the DTM data are probably high frequent angular movements of the spacecraft not sufficiently modeled in the bundle adjustment. The possibility of these oscillations in the cameras attitude are confirmed by members of the mission team. But their reason is still unknown. Because the frequencies of these oscillations are much higher than the used distance between the orientation points of $10 \mathrm{~s}$, this kind of systematic effect could not be modeled in the bundle adjustment and induces inconsistencies in the ray intersections.

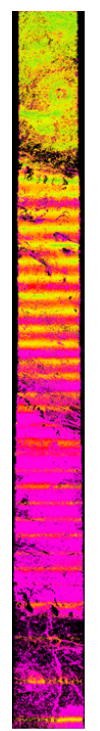

a)

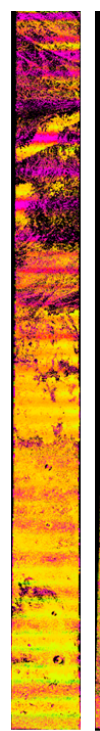

b)

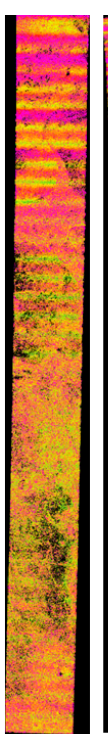

c)

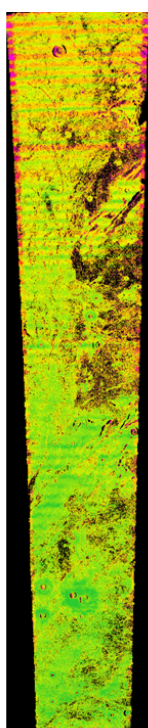

d)
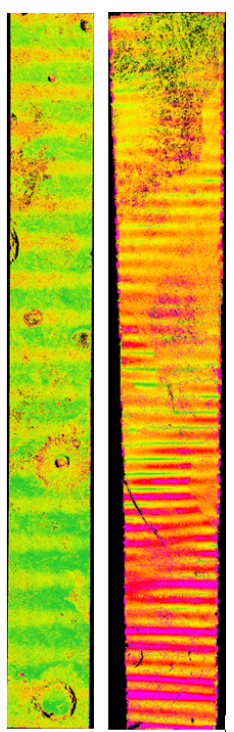

e)

f)
Figure 2: Orbits with remaining systematic errors after bundle adjustment (OPD $=10 \mathrm{~s})$. The orbit numbers are: a) h2028; b) h3079; c) h4311; d) h5081; e) h7222; f) h8393; green $=$ high, purple $=$ low geometric accuracy

One solution for this problem reconstructs the observed inconsistencies by minimizing the intersection error within a sequential adjustment procedure (Gwinner et al., 2010).

Another approach, presented here, is the consistent modeling of these oscillations directly inside the bundle adjustment by reducing the distance between the orientation points. Because the parameters of the exterior orientation between two orientation points are interpolated as described above, a shorter distance allows a better fit of the model to the actual camera orientation, as long as enough tie points are available. This method is now integrated into the standard processing work flow at IPI and led to new adjustment results as presented below.

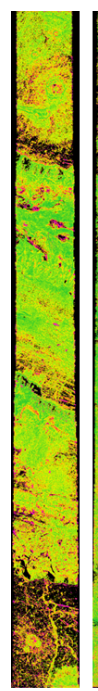

a)

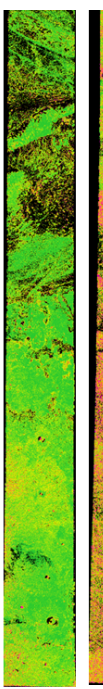

b)

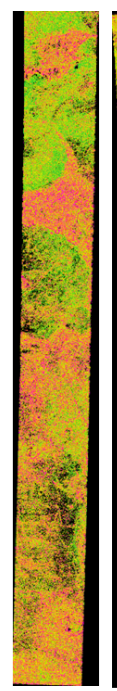

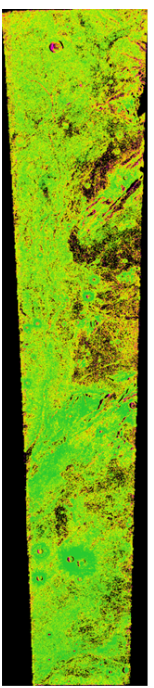

d)

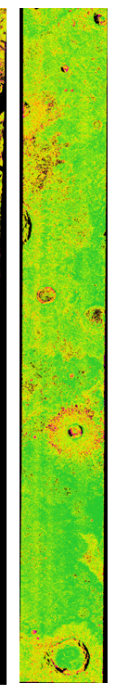

e)

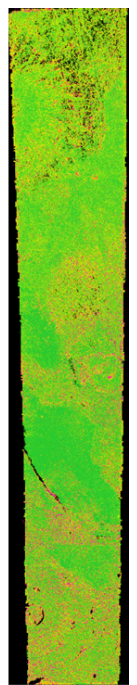

f)
Figure 3: The same orbits as in Figure 2 without systematic errors after bundle adjustment $(\mathrm{OPD}=2 \mathrm{~s})$; green $=$ high, purple $=$ low geometric accuracy (same color legend as in 2)

In Figure 2 the DTM point accuracy maps of six orbits are shown as an example. Pixels with green color indicate DTM points with 
high accuracy. Lower accuracies are shown by red, or even purple pixels. For more detailed information of these maps refer to the work about DTM generation (Gwinner et al., 2009).

As described in 2.6 the maps are created with an OPD of $10 \mathrm{~s}$, thus high frequent oscillations in the orientation data are visible in the quality of the DTM object points. Processing of these orbits with an OPD of $2 s$, as described above, leads to the quality maps shown in Figure 3. Expectedly the oscillations have been removed completely.

Reprocessing of the 299 orbits, where oscillations were found, succeeded in 232 cases. For about half of them, the adjustment resulted in DTM quality improvements comparable to the six example orbits (Figure 2 and 3). For the other orbits, the adjustment with an OPD of $2 s$ did not show an major improvement compared to an OPD of $10 \mathrm{~s}$. In 67 cases the bundle adjustment did not succeeded due to a lack of sufficient tie points.

Just as for the evaluation of the standard bundle adjustment results (see chapter 3 ) the mean intersection error was taken to reveal the improvement obtained by reducing the OPD.

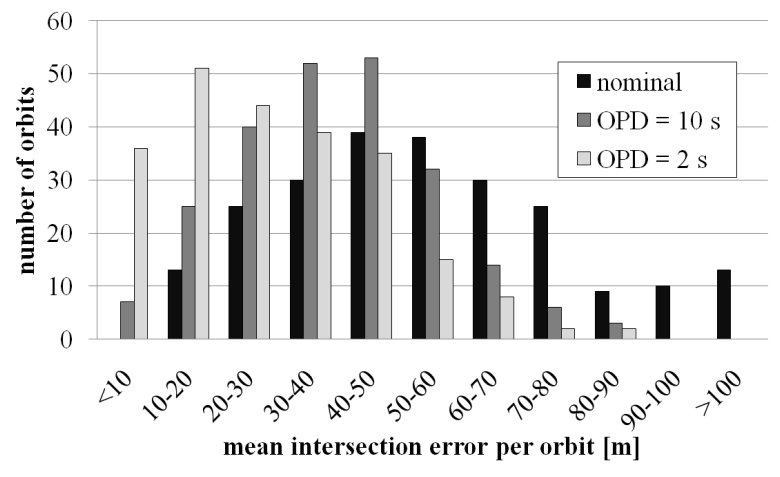

a) histogram

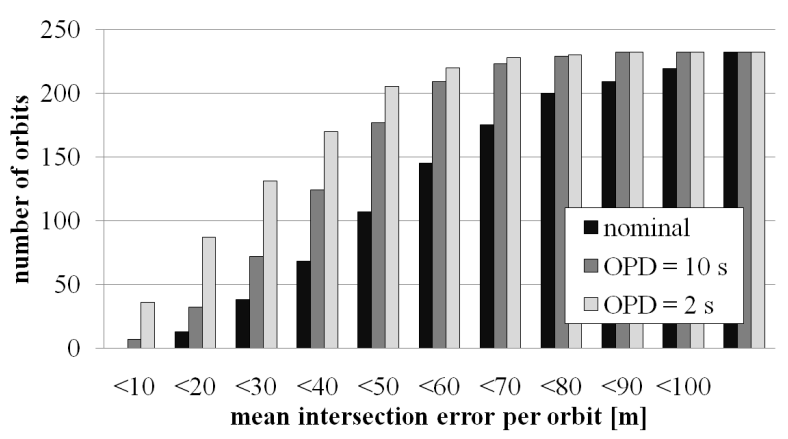

b) cumulative histogram

Figure 4: Number of orbits grouped by their mean intersection error before (black), after bundle adjustment with OPD $=10 \mathrm{~s}$ (gray) and $\mathrm{OPD}=2 s$ (light gray)

Figure 4 shows the mean intersection errors for the successfully processed 232 orbits. The quality of derived DTMs with nominal orientation data is displayed in black, after a bundle adjustment with an OPD of $10 s$ in gray, and with an OPD of $2 s$ in light gray. Compared to Figure 1 the quality of the nominal and the normally adjusted data is poorer (the peak of the histogram is in the middle of the graph). The improvement achieved by the standard bundle adjustment is about the same as for the entire data set with all orbits (see Table ?? and 3). When using the OPD of $2 s$ (light gray bars) the mean intersection errors are considerably smaller and the distribution of the orbits within the quality groups is as good as for the data without oscillations.

The relative improvement between the nominal and the adjusted orientation data is shown in the two following tables for a bundle adjustment with an OPD of $10 s$ in Table 3, respectively for $2 s$ in Table 4.

\begin{tabular}{|c||c|c|}
\hline Improvement & Nr. of Orbits & \% of 232 \\
\hline \hline $0.95-1.05$ & 20 & $8.6 \%$ \\
\hline $1.05-1.50$ & 131 & $56.5 \%$ \\
\hline $1.50-2.00$ & 55 & $23.7 \%$ \\
\hline $2.00-4.00$ & 25 & $10.8 \%$ \\
\hline$>4.00$ & 1 & $0.4 \%$ \\
\hline
\end{tabular}

Table 3: Factor of reduction of the mean intersection error (OPD $=10 \mathrm{~s}$ )

\begin{tabular}{|c||c|c|}
\hline Improvement & Nr. of Orbits & \% of 232 \\
\hline \hline $0.95-1.05$ & 109 & $47.0 \%$ \\
\hline $1.05-1.50$ & 40 & $17.2 \%$ \\
\hline $1.50-2.00$ & 28 & $12.1 \%$ \\
\hline $2.00-4.00$ & 38 & $16.4 \%$ \\
\hline$>4.00$ & 17 & $7.3 \%$ \\
\hline
\end{tabular}

Table 4: Factor of additional reduction of the mean intersection error (between $\mathrm{OPD}=10 s$ and $\mathrm{OPD}=2 s$ )

It should be noted that the factors of improvement in Table 4 are additional to the improvement already obtained via standard bundle adjustment (with OPD $=10 \mathrm{~s}$ ). Thus the increase compared to the nominal orientation data is clearly higher. The number of orbits where the change of the mean intersection error is lower than $5 \%$ is 109 . This fact can be explained with a concept used in the adjustment process. If the number of tie points in an orbit is too small, either locally or over the entire strip, for a stable determination of the parameters of exterior orientation, the OPD is automatically increased by the used software, so that the adjustment becomes more stable. This impedes the modeling of the high frequent oscillations, so the error of the intersections could not be reduced. For the HRSC images of 123 orbits reprocessing of the data via bundle adjustment with an OPD of $2 s$ leads to a relevant reduction of the mean ray intersection error.

\section{CONCLUSIONS AND FUTURE WORK}

From the 2535 investigated HRSC orbits, the exterior orientation of $91.9 \%$ (2329 orbits) has been improved using bundle adjustment. This improvement minimizes the residuals of generated DTMs points and also provides a high consistency between the HRSC object points and the MOLA DTM. It has been shown in this paper, that for a number of 123 orbits further improvements of the exterior orientation can be achieved by reducing the orientation point distance, to allow a more precise modeling of the Mars Express trajectory.

To increase the number of orbits, where the bundle adjustment succeeds with good results, further enhancements of the bundle adjustment procedure are necessary. For orbits without enough tie points, a potential approach for improving the adjustment of the exterior orientation, is to vary the distance between the orientation points, both over time and with separate values for the six elements of the exterior orientation. In this way the trajectory of the spacecraft can be reconstructed more stable even if the image information is weak. 


\section{ACKNOWLEDGEMENTS}

We thank the HRSC Experiment Teams at DLR Berlin and Freie Universität Berlin as well as the Mars Express Project Teams at ESTEC and ESOC for their successful planning and acquisition of data as well as for making the processed data available to the HRSC Team. This work is funded by the Deutsches Zentrum für Luft- und Raumfahrt e.V. (DLR) under grant no. 50 QM 0902. This support is gratefully acknowledged.

\section{REFERENCES}

Dorrer, E., Maier, W. and Uffenkamp, W., 1995. Analytical kinematic sensor orientation of MOMS-02 linear stereo imagery. In: Colomina/Navarro (eds.): Integrated Sensor Orientation. pp. 261-273.

Dumke, A., Spiegel, M., Schmidt, R., Michael, G. and Neukum, G., 2008. Mars: High resoluition digital terrain model and ortho-image mosaic on the basis of MEX/HRSC data. International Archives of Photogrammetry and Remote Sensing 37(4), pp. 1037-1042.

Gwinner, K., Scholten, F., Preusker, F., Elgner, S., Roatsch, T., Spiegel, M., Schmidt, R., Oberst, J., Jaumann, R. and Heipke, C., 2010. Topography of Mars from global mapping by HRSC highresolution digital terrain models and orthoimages: Characteristics and performance. Earth and Planetary Science Letters 294(3-4), pp. 506-519.

Gwinner, K., Scholten, F., Spiegel, M., Schmidt, R., Giese, B., Oberst, J., Heipke, C., Jaumann, R. and Neukum, G., 2009. Derivation and Validation of High-Resolution Digital Terrain Models from Mars Express HRSC-Data. Photogrammetric Engineering \& Remote Sensing 75(9), pp. 1127-1142.

Hofmann, O., Nave, P. and Ebner, H., 1982. DPS - A Digital Photogrammetric System for Producing Digital Elevation Models and Orthophotos by Means of Linear Array Scanner Imagery. International Archives of Photogrammetry and Remote Sensing 24 (3), pp. 216-227.

Kornus, W., 1999. Dreidimensionale Objektrekonstruktion mit digitalen Dreizeilenscannerdaten des Weltraumprojekts MOMS02/D2. Dissertationsschrift, DGK-C, Nr. 496, Deutsche Geodätische Kommission, München.

Müller, F., 1991. Photogrammetrische Punktbestimmung mit Bildern digitaler Dreizeilenkameras. Dissertationsschrift, DGKC, Nr. 372, Deutsche Geodätische Kommission, München.

Neukum, G., Jaumann, R. and the HRSC Co-Investigator Team, 2004. HRSC: The High Resolution Stereo Camera of Mars express. Mars Express: The Scientific Payload, Eur. Space Agency Spec. Publ., ESA-SP 1240, pp. 17-36.

Ohlhof, T., 1996. Lokale, regionale und globale Punktbestimmung mit Dreizeilenbilddaten und Bahninformationen der Mars 96-Mission. Dissertationsschrift, DGK-C, Nr. 445, Deutsche Geodätische Kommission, München.

Schmidt, R., Spiegel, M., Heipke, C., Dumke, A., Neukum, G. and the HRSC Co-Investigator Team, 2008. Operational Determination of Tie Points and Bundle Adjustment of HRSC Images of the Mars Express Mission. International Archives of Photogrammetry and Remote Sensing 37(4), pp. 1025-1030.

Spiegel, M., 2007a. Improvement of Interior and Exterior Orientation of the Three Line Camera HRSC with a Simultaneous Adjustment. International Archives of Photogrammetry and Remote Sensing 36(3/W49B), pp. 161-166.

Spiegel, M., 2007b. Kombinierte Ausgleichung der Mars Express HRSC Zeilenbilddaten und des Mars Global Surveyor MOLA DGM. Dissertationsschrift, DGK-C, Nr. 610, Deutsche Geodätische Kommission, München.
Strunz, G., 1993. Bildorientierung und Objektrekonstruktion mit Punkten, Linien und Flächen. Dissertationsschrift, DGK-C, Nr. 408, Deutsche Geodätische Kommission, München. 